\title{
NEW MAGNETIC FILTER FOR CHEMICAL INDUSTRY
}

\author{
I. SPEVAKOVA ${ }^{*}$, L. RUDITSER ${ }^{\dagger}$, V. EROSHENKO ${ }^{\dagger}$ and \\ G. NICKOLSKIY ${ }^{\dagger}$ \\ * Technion, Faculty of Civil Engineering, Haifa 32000, Israel \\ $\dagger^{\dagger}$ Ukrainian Institute of Civil Engineering, Kharkov, Ukraine
}

(Received February 9, 1993, revised April 29, 1993)

\begin{abstract}
An economical, inexpensive and compact magnetic filter with pulse magnetization and demagnetization of the filtering matrix, consuming no electric power throughout the process was constructed and its effectiveness was tested with micron-sized magnetite and Raney nickel catalyst. Complete filtration of $+1 \mu \mathrm{m}$ particles was achieved at rate $\leq$ $150 \mathrm{~m} / \mathrm{h}$. The proposed filter can be of use in chemical industry for the recovery of micron-sized magnetic particles from various liquors.
\end{abstract}

\section{INTRODUCTION}

Many processes of chemical synthesis (hydrogenation, dehydrogenation, hydrogenolysis, isomerization etc.) are carried out in the presence of catalysts, in which capacity the transition metals $\mathrm{Fe}, \mathrm{Ni}$, Co and their alloys, oxides and other compounds are widely used [1]. Their catalytic activity is due to partially filled $\mathrm{d}$-orbitals. It has been recently shown that some compounds of rare-earth elements with partially filled f-orbitals can also serve as catalysts $[2,3]$.

When the chemical catalytic reaction takes place in a liquor, the catalyst is generally introduced in dispersed and suspended form, because of strong 
dependence of its activity on the area of the contact surface. Small-sized pellets satisfy this requirement to a large degree. However, upon completion of the reaction a problem of the catalyst recovery arises. High efficiency of the recovery is essential with a view both to ensure purity of the product and to minimize losses of expensive catalyst components.

Large catalyst pellets are recoverable by precipitation, mechanical filtration or centrifugation techniques [4], but fine particles, either used intentionally, or formed by attrition from larger ones present a more difficult task. Mechanical (cloth) filter cannot provide complete separation of micron-sized material and is subject to heavy clogging with the attendant prohibitive labour and time requirements.

Thanks to their strong magnetic properties, the transition and rare-earth metals and their compounds are recoverable by magnetic filtration. The filtering matrix with its relatively open structure offers a limited resistance to the moving liquid. The efficient separation is thus achieved at high flow velocities, without undue pressure drops and the captured contaminants can be discharged by backflushing without the matrix having to be opened. The magnetic filtration technique is suitable even for the removal of submicron ( 0.1 to $1 \mu \mathrm{m})$ paramagnetic particles $[5,6]$ from suspensions.

Design of a magnetic filter depends on magnetic properties and the size of the material involved. Naturally, strong magnetic fields and high field gradients are required for the filtration of weakly paramagnetic submicron particles and complex and expensive separators are used. In the sequel, when speaking of heterogeneous magnetic catalyst we refer to micron-sized particles (generally 1 to $100 \mu \mathrm{m}$ ) which are often ferro- or ferrimagnetic, and for which no such an apparatus is needed.

In this work we present a design of a simple, low-cost and very economical magnetic filter. Its operating characteristics were investigated with a view of possible application in the removal of heterogeneous nickel catalyst from liquors. Some of these characteristics were studied on fine magnetite $\left(\mathrm{Fe}_{3} \mathrm{O}_{4}\right)$ powder, a highly suitable material due to its accessibility and low cost.

In addition filtration of fine magnetite particles can be considered to be a separate task because of the fact that undesired contaminants of different industrial fluids 
(ammonia water, steam condensate, cooling water) were found to contain magnetite as the most frequent component resulting from equipment corrosion or were formed during thermo-mechanical treatment of steel parts. for example $[5,7]$, in the manufacture of nitric acid the problem of magnetite penetrating the reactor with the ammonia from pipelines and tank cars and poisoning the platinum-alloy catalyst was reduced my a magnetic filter installed in the liquid ammonia feedline resulting in a significant increase of nitric acid yield and of lifetime of the catalyst. It is also noteworthy that nonmagnetic catalysts can be prepared on magnetite-containing substrates, as was reported in [8].

\section{CHARACTERISTICS OF REMOVED MATERIALS}

A sample of fine nickel catalyst, from an application which involved a problem of its removal from the waste methanol solution, was investigated. A thick suspension of the catalyst was received from the plant and dried at $110^{\circ} \mathrm{C}$ for examination of its magnetic and other physical properties, relevant to the filtration process. Magnetic filtration investigations were conducted on suspensions with appropriate concentrations prepared by suspending the dry powder in the water. The catalyst was prepared at the plant by grinding the $\mathrm{Ni}-\mathrm{Al}$ alloy and leaching out aluminium with soda. The magnetite powder consisted of ground ore.

Table I presents characteristic granulometric compositions of both powders, obtained using a "Special PMC" device. Weight concentrations were calculated on the assumption of spherical particles. Densities of the powders measured by pycnometric technique are given in Table II with pure nickel included for comparison. It can be seen that the extraction of $\mathrm{Al}$ from the catalyst pellets was only partial.

Magnetic properties of alloys can differ significantly from those of pure metals. They depend on the composition of the alloy and on its thermal, mechanical or magnetic treatment. In powdered samples, particle size is of great importance. Ferromagnetic powder samples have a coercive force substantially higher than the corresponding bulk material, and their magnetization curves are less steep. 
Table I. Granulometric composition of powders

\begin{tabular}{lcccc}
\hline \multirow{2}{*}{$\begin{array}{c}\text { Size, } \\
\mu\end{array}$} & $\begin{array}{l}\text { Raney nickel } \\
\text { size fraction }\end{array}$ & $\begin{array}{l}\text { Magnetite } \\
\text { centration, } 8\end{array}$ & $\begin{array}{l}\text { Numerical } 8 \text { of } \\
\text { size fraction }\end{array}$ & $\begin{array}{c}\text { Weght con- } \\
\text { centration, } 8\end{array}$ \\
\cline { 3 - 5 } $0.3-0.5$ & 0.0 & 0 & 3 & 0.002 \\
$0.5-1.0$ & 0.2 & 0.00003 & 17 & 0.087 \\
$1.0-2.0$ & 5.5 & 0.0077 & 38 & 1.557 \\
$2.0-4.0$ & 19.2 & 0.2158 & 28 & 9.180 \\
$4.0-8.0$ & 37.7 & 3.3897 & 10 & 26.228 \\
$8.0-16$ & 27.6 & 19.8519 & 3 & 62.946 \\
$16-32$ & 9.3 & 53.5161 & 0 & 0.0 \\
$32-64$ & 0.5 & 23.0177 & 0 & 0.0 \\
\hline
\end{tabular}

Table II. Comparative properties of powdered samples

\begin{tabular}{lcc}
\hline Material & $\begin{array}{c}\text { Density, } \\
\mathrm{g} / \mathrm{cm}^{3}\end{array}$ & $\begin{array}{c}\text { Dynamic magnetic suscept., } \\
\text { CGSM/g, for packing } \tau=0,15\end{array}$ \\
\hline Raney nickel & 4.5 & $6.2310^{-2}$ \\
Pure nickel & 8.8 & $7.3310^{-2}$ \\
Natural magnetite & 4.6 & $8.6010^{-2}$ \\
\hline
\end{tabular}

Nickel-aluminium alloys are known to be ferromagnetic or nonmagnetic. The $\alpha$-solution is ferromagnetic at room temperature and contains up to $5 \% \mathrm{Al}$, its magnetization decreases almost linearly with increasing Al content [9]. Magnetic properties of powdered samples of Raney nickel catalyst were investigated in [10] and it was shown that the catalyst pellets containing less than $5 \% \mathrm{Al}$ (implying the formation of the $\alpha$-phase) can be obtained by prolonged leaching. Powders of natural magnetite contain generally such impurities as $\mathrm{FeO}, \mathrm{Fe}_{2} \mathrm{O}_{3}$, quartz, martite etc., and their magnetic field behaviour depends on the particular ore deposit. 
Table III. Some magnetic properties of powders ${ }^{*}$

\begin{tabular}{|c|c|c|c|c|c|}
\hline Compound & $\begin{array}{l}\text { Curie } \\
\text { point, }{ }^{\circ} \mathrm{C}\end{array}$ & $\begin{array}{l}\text { Saturation } \\
\text { induction, } \\
4 \pi I_{a}, G s\end{array}$ & $\begin{array}{l}\text { Residual } \\
\text { induction, } \\
4 \pi I_{R} \text {, Gs }\end{array}$ & $\begin{array}{c}\text { Coercive } \\
\text { force, } \\
\mathrm{H}_{x}, \mathrm{Oe}\end{array}$ & Refer. \\
\hline $\begin{array}{l}\text { Ni-Al alloy } \\
\text { (bulk): }\end{array}$ & & & & & \\
\hline $2.56 \% \mathrm{Al}$ & & 3915 & & & {$[9]$} \\
\hline $\begin{array}{l}\quad 4.7 \% \mathrm{Al} \\
\text { Raney nickel } \\
\text { (powder): }\end{array}$ & 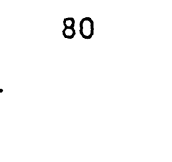 & 2237 & & & [9] \\
\hline $2.56 \% \mathrm{Al}$ & & 4362 & 1221 & 230 & {$[10]$} \\
\hline $4.7 \& \mathrm{Al}$ & & 3355 & 1007 & 190 & {$[10]$} \\
\hline $\mathrm{Ni}$ (bulk) & 358 & 6084 & 4000 & 1 & {$[9,11]$} \\
\hline $\mathrm{Ni}$ (powder) & & & & 200 & {$[9]$} \\
\hline $\begin{array}{l}\text { Magnetite } \\
\text { (bulk) }\end{array}$ & 575 & 6150 & 5000 & 600 & {$[9,11]$} \\
\hline $\begin{array}{l}\text { Magnetite } \\
\text { (powder) }\end{array}$ & & 4000 & 1500 & $20-150$ & [12] \\
\hline
\end{tabular}

As regards the Raney nickel, its main properties, as reported in literature, are listed in Table III, including the corresponding properties of pure nickel. The data in the Table indicate a similarity in behaviour of the powders in the magnetic field, and their dynamic magnetic susceptibility is a convenient criterion for comparison.

The corresponding data shown in Table II were obtained in the magnetic field up to 4.7 Oe with the aid of the E7-2 low-frequency induction gauge (Soviet manufacture). The pure nickel powder has a mean particle size of $50 \mu \mathrm{m}$. The magnetic susceptibility and magnetization pattern of the disperse sample depend on their porosity and the measured value of susceptibility increases with 
packing [5]. Measurements of susceptibility were conducted on samples of the same packing level, $\tau=0.15$. (Although the $\tau<0.015$ to 0.02 range suffices for these measurements from the viewpoint of mean particle spacing, the above higher level was used because of sample-settling considerations).

\section{DESIGN OF A MAGNETIC FILTER}

Magnetic filters are conveniently classified in three groups according to their magnetic systems: (a) - permanent; (b-c) - electromagnet (coil-type) with yoke and without it. Permanent magnets are advantageous from the energy consumption point of view, but they complicate the backwashing problem which usually necessitates the removal of the matrix or the application of powerful liquid or air jets. Electromagnetic filters generate considerably stronger magnetic field and simplify the regeneration process but entail large energy consumption throughout the working cycle.

The filter under discussion combines low energy consumption characteristics of the permanent magnet with advantages of the electromagnet. Its basic scheme is shown in Fig. 1. Canister 1, made of nonmagnetic stainless steel, is charged with the filtering matrix 2 , consisting of ferromagnetic, magnetically soft elements. The magnetically hard core 3 is magnetized by current pulses that pass through the coil 5 and play the role of the permanent magnet in the filtration process. The magnetic circuit is closed through the yoke 4 made of a magnetically soft material and of the filtering matrix, thereby obviating the effect of demagnetizing field and ensuring maximum possible residual magnetization for the core material to improve the filtration regime.

Backwash current pulse of opposite polarity, proportional to the coercive force of the core material, is passed resulting in demagnetization of the core and of the matrix. In these circumstances the magnetic field does not interfere with the wash-out operation.

The backwash of the filter is not a simple problem and it was found that zero magnetic field does not suffice, even under intensive supply of the washing water, 
for regeneration of the matrix whose elements undergo cementation after several cycles. To overcome this phenomenon, mechanical loosening, pulse washing water intensity or combined water-air backwash are used, with attendant complication of the design of the filter and an increase in its cost.

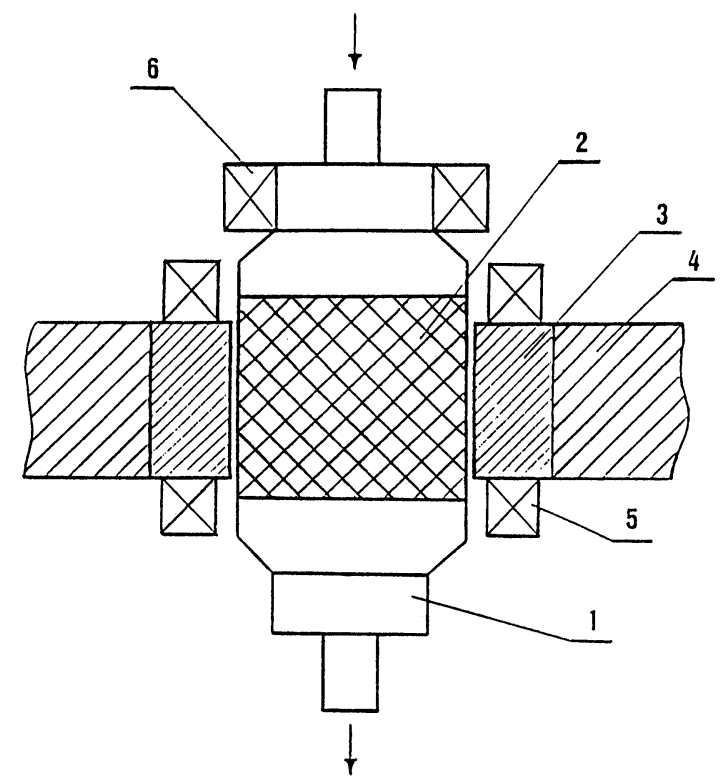

Fig. 1. Schematic diagram of the magnetic filter: 1-canister, 2-filtering matrix, 3-magnetically hard core, 4-yoke, 5-magnetizing coil, 6-agitation pulse solenoid.

Our solution of the problem consisted in agitating the matrix by means of a magnetic force induced by the electric current pulses in a special coil 6 - the agitating solenoid, arranged around the upper part of the canister, clear of the matrix, and fed by a generator of low-frequency pulsating voltage. With the pulse on, the gradient of the magnetic field entrains the elements of the matrix into the upper empty space of the canister and at the end of the pulse they drop back by gravity. The regeneration takes place after the demagnetization of the system and consists in passing the steam of water through the matrix in conjunction with its periodic agitation as above. The matrix is re-packed to the working level in the following filtration cycle during the magnetization stage. 
As the upper layers of the matrix are agitated more efficiently than the lower ones, the effect on the backwash is more pronounced when the filtration proceeds in the downward direction (the upper layers being more intensively clogged) and the backwash proceeds upwards. Such a geometry is not always easy to realize but it is optimum for a ball matrix. When the matrix consists of oblong shavings, the solenoid can be located at the bottom of the separator and its downward-directed magnetic force makes for lengthwise vertical arrangement of the elements. In such a case the filtration and the backwash can proceed upwards and downwards, respectively.

\section{OPERATING CHARACTERISTICS OF THE FILTER}

In the laboratory model the $\mathrm{Fe}-\mathrm{Ni}-\mathrm{Al}-\mathrm{Co}$ alloy with residual magnetic induction of $B_{R} \cong 11 \mathrm{kG}$ and coercive force $\mathrm{H}_{\mathrm{C}} \cong 450$ Oe was used as the core material. The spacing of the poles was $120 \mathrm{~mm}$, the magnetic field induced in the space between the poles, with the matrix absent, was $\cong 500$ Oe. The maximum dimensions of the filter, including the yoke, were $0.4 \times 0.4 \times 0.7 \mathrm{~m}$ and its mass, including the matrix, was less than $100 \mathrm{~kg}$.

The material variants of the matrix were the balls with diameter $d=4.0 \mathrm{~mm}$, made of ordinary steel (type ShKh15) and the shavings of stainless magnetically soft steel (40Kh13). The magnetic field induction achieved with these variants (as measured in $15 \mathrm{~mm}$ slit gap) are shown in Table IV, column 3. Both steel elements were magnetically soft and could be magnetized in the field $H \cong 10 \mathrm{kOe}$ up to $B \cong 13 \mathrm{kG}$ (for porous media [12], as against $B \cong 22 \mathrm{kG}$ for non-porous substances). The differences in the magnetic induction values, as seen in Table IV are mostly attributable to differences in porosity, depending on the shape and dimensions of the shavings (packing values up to $\tau \cong 0.55$ can be reached owing to their disintegration). With ball elements the packing does not depend on their diameter.

It can be seen that substantially higher field can be achieved through increased packing but it is not always useful. For sufficiently large ferromagnetic particles it can be preferable to increase the loading capacity of the matrix and the duration of 
the cycle. Thus the possibility of varying the porosity is a great advantage of the shavings apart from their low cost as a scrap material.

It is also noteworthy that the magnetic induction in the pores of the balls made of non-stainless steel (because of price consideration) decreases after prolonged exploitation because of the formation of a nonmagnetic oxide film on the ball surfaces. The can be rectified, for instance, by pre-coating the balls with a nickel ferromagnetic film.

Table IV. Magnetic field induction generated in the separator with different matrices

\begin{tabular}{lccc}
\hline Matrix & Packing & $\begin{array}{c}\text { Induction in } \\
\text { 15m slit gap, Gs }\end{array}$ & $\begin{array}{c}\text { Induction at } \\
\text { Hopr }=2 \text { kOe [12] }\end{array}$ \\
\hline $\begin{array}{l}\text { Steel balls } \\
\text { (ShKh15) }\end{array}$ & 0.6 & 9100 & 6700 \\
$\begin{array}{c}\text { Stainless steel } \\
\text { shavings (40Kh13) } \\
-"-\end{array}$ & 0.33 & 6600 & 5500 \\
& 0.23 & 5200 & 4200 \\
\hline
\end{tabular}

For the electromagnetic filters used in industry for the removal of magnetic impurities from liquids and gases [12], the level of electric current corresponding to the magnetizing field of $\mathrm{H} \cong 10 \mathrm{kOe}$ is impractical as the rate of increase of the magnetic induction drops steeply with an increase of the external magnetic field, and the $\mathrm{H} \cong 1$ to $2 \mathrm{kOe}$ range can be considered as optimum. The magnetic induction corresponding to this optimum field in porous matrices of the same material and packing (again according to [12]) is listed in the right-hand column of Table IV and it can be seen to be substantially lower.

Stronger field is important for filtration of small and weakly magnetic particles and its advantage is demonstrated by our results given in Table $\mathrm{V}$, for the ball matrix and a suspension of magnetite. 
Table V. The dependence of the quality of the filtrate on the magnetic field induction in the matrix

\begin{tabular}{crc}
$\begin{array}{c}\text { Strength of } \\
\text { magnetizing pulse, } V\end{array}$ & $\begin{array}{c}\text { Induction in } \\
15 \mathrm{~mm} \text { slit gap, Gs }\end{array}$ & $\begin{array}{c}\text { Filtrate concentration, } \\
\mathrm{mg} / \mathrm{l}\end{array}$ \\
\hline 400 & 9100 & 1.5 \\
240 & 4800 & 8.2 \\
180 & 2500 & 76 \\
\hline
\end{tabular}

The magnetic field was varied through the strength of the magnetizing pulse. Magnetite with a large fraction of small particles was separated from a suspension with initial concentration $1 \mathrm{~g} / \ell$, with the filtration rate of $125 \mathrm{~m} / \mathrm{h}$. Comparison with granulometric composition of the magnetite powder (Table I) shows that the optimum filtrate concentration under the strongest magnetization of the core was obtained with particles up to the minimum size of approximately $1 \mu \mathrm{m}$.

The energy consumption of our filter is 2 Wh for a 30 minute cycle ( 4 pulses per hour). Simple calculation shows that this consumption level is about 500 times lower than that of a direct current coil generating the magnetic field of $2 \mathrm{kOe}$ in the space of the same volume. Thus, with a magnetically hard core serving as poles of a system with closed magnetic circuit, the efficiency of the filtration process can be improved at reduced energy consumption.

The advantage of the proposed method of regeneration was confirmed by our experiment (Figure 2), in which the backwash was performed either with or without agitation (curves 1 and 2, respectively). In both cases the matrix was of the ball type and the material removed was magnetite. The filtration yield was $6 \%$ of the matrix by weight, the rates of filtration and backwash were $125 \mathrm{~m} / \mathrm{h}$ and $200 \mathrm{~m} / \mathrm{h}$, respectively. It can be seen that with the same amount of washing water the backwash efficiency under agitation was increased by 30 to $40 \%$, reaching nearly $100 \%$. Variation of the agitation frequency in the 0.5 to $1.0 \mathrm{~Hz}$ interval did not produce significant changes. 
It should be noted that considerable improvement of the matrix regeneration is achieved by the adjustment of the $\mathrm{pH}$ of the washing water [13]. This procedure is efficient for magnetic filtration of waste water but may not be acceptable for technological solutions of high-purity requirement.

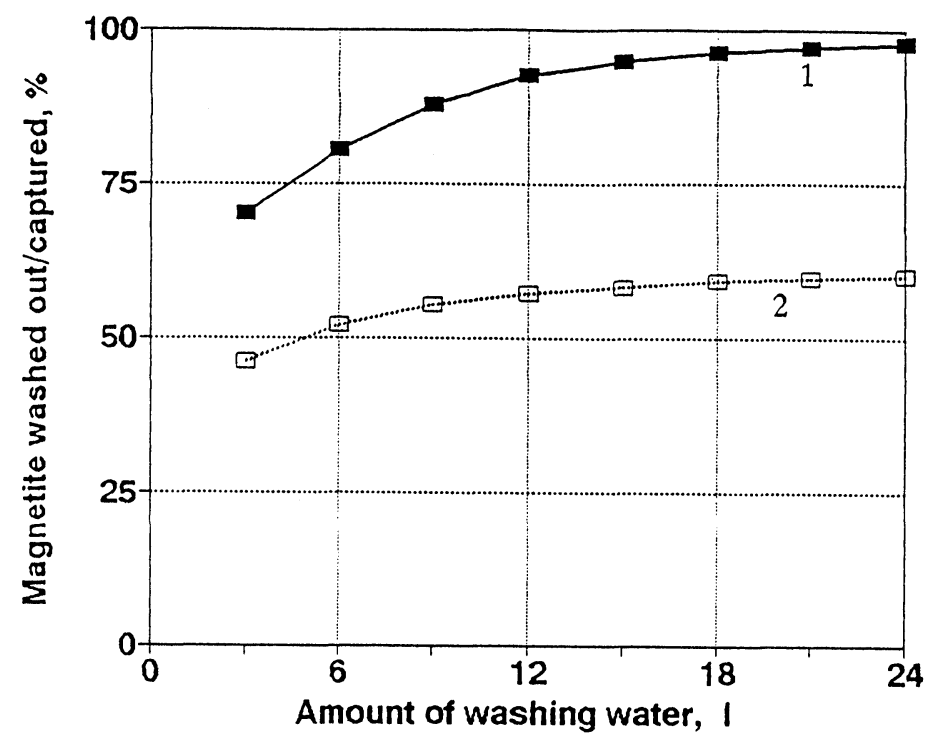

Figure 2. The effect of agitation on the backwash: 1 - with agitation, 2 - without agitation

\section{THE CHARACTERISTICS OF THE FILTRATION}

Sedimentation tests and microscopic observations under the magnetic field showed that Raney nickel powder used in the study contained a lighter non-magnetic fraction (unleached aluminium). This ruled out the evaluation of the quality of the filtrate (the amount of magnetic particles that were not captured) by turbidity measurements. An alternative solution is X-ray radiography of sediments obtained by passing the magnetic filtrate through a paper filter. This expensive method, however, failed to yield sufficient information, and, subsequently, the study was carried out using a series of artificial suspensions of magnetite. 
The ball matrix proved to be uneconomical for highly concentrated $(>1 \mathrm{~g} / \ell)$ suspensions because of the attendant strong clogging (of the silt-deposition type). By contrast, in the case of the more porous shaving matrix the clogging effect was moderate and silting-free, and a similar result was obtained with the ball matrix after separation of a heavy fraction from the magnetite suspension by elutriation.

Figure 3 shows the turbidity of the filtrate versus the filtration rate, obtained for the $1 \mathrm{~g} / \ell$ suspension. All data, according to the granulometric composition, correspond the particles up to the minimum size of approximately $1 \mu \mathrm{m}$. Turbidity can be seen to increase with the initial concentration as a result of the increase in uncaptured submicron particles. In the case of Raney nickel, no nickel crystallites above $1 \mu \mathrm{m}$ were observed in the $\mathrm{X}$-ray photographs of sediments after the filtration through the matrix of the shavings of both porosities, at rates below $150 \mathrm{~m} / \mathrm{h}$.

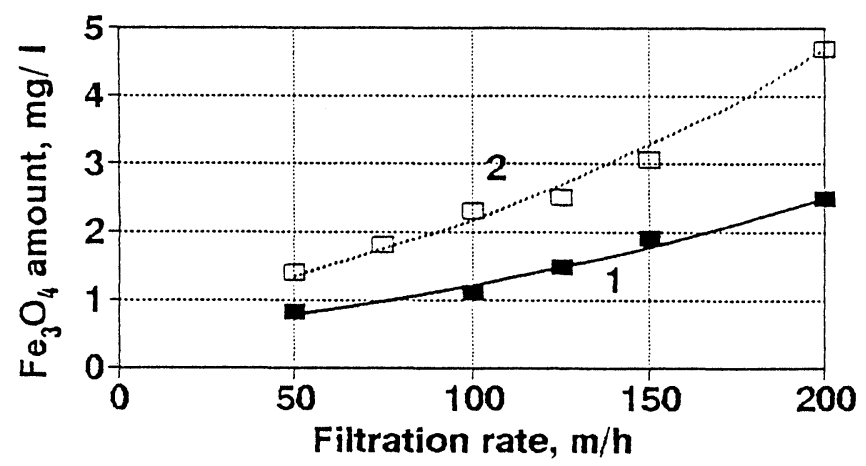

Figure 3. The concentration of magnetite in the filtrate versus filtration rate for different types of matrix elements: 1 - steel balls with $\tau=0.6,2-$ stainless steel shavings with $\tau=0.23$. The initial concentration $1 \mathrm{~g} / \ell$.

The initially established filtration rate drops as the matrix loading increases (Figure 4): the lower the porosity, the steeper the drop. For the ball matrix of $\tau=0.6$, the curve corresponds to that for the light fraction. For the matrix of the shavings, no significant changes were observed after the removal of heavy fraction. The quality of the filtrate improves slightly with the increase of the matrix loading 
and the corresponding filtration rate decreases. The loading capacities of the matrices can be taken as $0.1 \mathrm{~kg}, 0.5 \mathrm{~kg}$ and $1 \mathrm{~kg}$ for $\tau=0.6,0.33$ and 0.23 , respectively, and the corresponding cycle duration at the initial concentration of $1 \mathrm{~g} / \ell$ and the filtration rate of $125 \mathrm{~m} / \mathrm{h}$ are $3.3,16.6$ and 33.3 minutes, respectively. This result, combined with the silting effect, demonstrates the inefficiency of the ball matrix for suspensions of $1 \mathrm{~g} / \ell$ ore more. This matrix is best used as the second stage, where it can improve the quality of the filtrate by a factor of two (Figure 3).
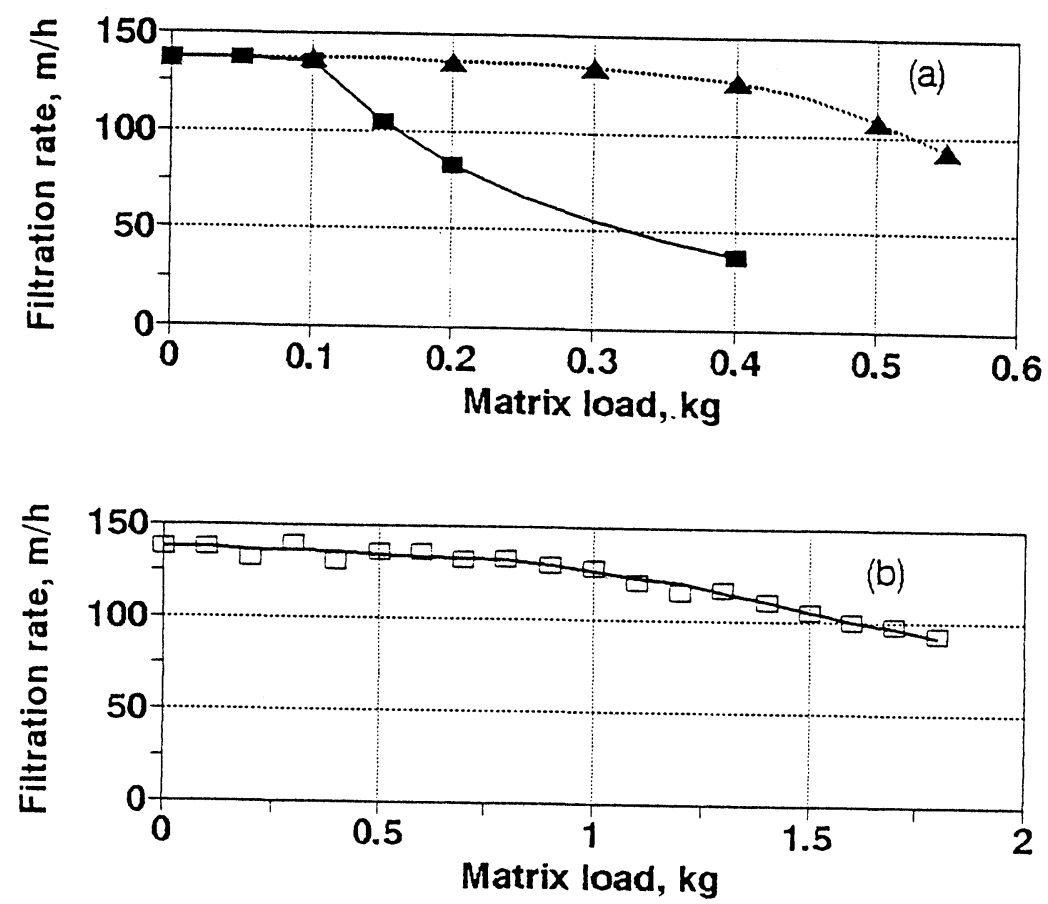

Figure 4. The filtration rate versus matrix loading, at different packing densities: (a) $\tau=0.6, \boldsymbol{\Delta} \tau=0.33$; (b) $\quad \tau=0.23$.

For suspensions of about $5 \mathrm{~g} / \ell$, the filtration cycle can be prolonged for all types of matrices, by preliminary separation of the heavy fraction of relatively large particles, with magnetic filtration serving as the second stage, exclusively for low-micron fraction. Particles above $10 \mu \mathrm{m}$ are readily separated by ordinary sedimentation in a settling tank. With the aid of Stoke's equation, the 
sedimentation rate of $10 \mu \mathrm{m}$ Raney nickel in water, density $4.5 \mathrm{~g} / \mathrm{cm}^{3}$, is found to be as high as $12.6 \mathrm{~m} / \mathrm{h}$ (for heavier particles or substances with density and viscosity less than those of water, it is even higher). After the separation of the $+10 \mu \mathrm{m}$ fraction, the initial concentration, according to the granulometric composition, drops by a factor of 10 and the cycle is prolonged proportionately.

A characteristic dependence of the backwash quality on the flow rate of the washing water is shown in Fig. 5 , for the matrix of the shavings of $\tau=0.23$, after the filtration of the suspension of Raney nickel, at the rate of $125 \mathrm{~m} / \mathrm{h}$, up to the level of the loading of $1 \mathrm{~kg}$. The recommended flowrates of the washing water are the same as, or higher than those used during the filtration.

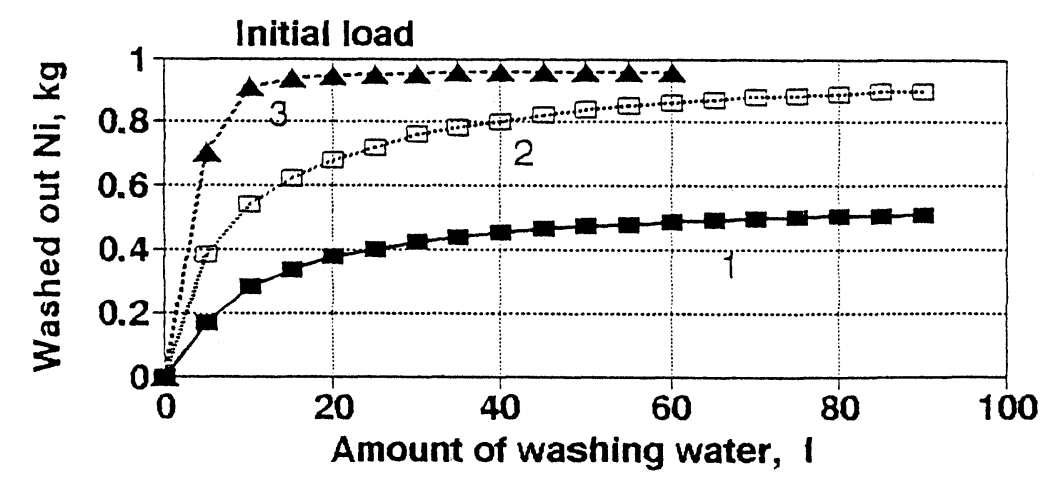

Figure 5. The backwash quality versus the flowrate of the washing water, at the packing density of $\tau=0.23$, the initial loading $1 \mathrm{~kg}$, filtration rate $125 \mathrm{~m} / \mathrm{h}: 1-50 \mathrm{~m} / \mathrm{h}, 2-125 \mathrm{~m} / \mathrm{h}, 3-$ $200 \mathrm{~m} / \mathrm{h}$.

\section{CONCLUSIONS}

1. An economical, low-cost and compact magnetic filter with pulse magnetization and demagnetization of the filtering matrix is suitable for the removal of weak ferromagnetic particles up to the minimum size of $1 \mu \mathrm{m}$, from liquid suspensions. The backwash efficiency is improved by agitation of the matrix elements. 
2. The yield of the laboratory filter at the filtration rate of $150 \mathrm{~m} / \mathrm{h}$, ensuring the removal of low-micron particles, was $0.6 \mathrm{\ell} / \mathrm{s}$, subject to the quality of the material available for preparation of the cores serving as poles of the magnetic system. With stronger magnetic materials [14], the yield can be increased.

3. In catalytic processes involving magnetically active elements, the magnetic filter can be used both in the final stage for extraction of heterogeneous catalyst particles from a liquor and in the preliminary stage for separation of catalytically active magnetic particles from inactive nonmagnetics introduced during the preparation process of the catalyst (as is the case of Raney nickel).

\section{ACKNOWLEDGMENT}

The authors wish to thank Professor Israel Lin for his helpful discussions and valuable remarks.

\section{REFERENCES}

1. Kirk-Othmer: Encyclopedia of Chemical Technology J. Wiley Interscience, 1979

2. Q. Shuncherg et al.: In: Proc. First Internatl. Conf. Metallurgy and Materials Science of Tungsten, Titanium, Rare Earths and Antimony, Pergamon Press, 1989, p. 1187

3. J. Yingtai et al.: Ibid., p. 366

4. T.A. Malinovskaya et al.: Separation of Suspensions in Chemical Industry, (in Russian), Khimiya, Moscow 1983

5. A.V. Sandulyak: Magnetic Filtration Purification of Liquids and Gases (in Russian), Khimiya, Moscow 1988

6. R.R. Birrs and M.R. Parker: In: Progr. Filtrat. Separ. 2 (1981), 171 
7. G.R. Gillespic and D. Goodfellow: Chem. Eng. Progress 70 (1974), 81

8. G.M. Whitesides et al.: Ind. Eng. Chem., Process Des. Dev. 15 (1976), 226

9. R.M. Bozorth: Ferromagnetism, Van Nostrand, New York 1951

10. G.A. Martin and P. Fouilloux: J. Catalysis 38 (1975), 231

11. Tables of Physical Values,(in Russian), Atomizdat, Moscow 1976

12. A.V. Sandulyak and V.I. Grashchenko: Electromagnetic Filters-Precipitators (in Russian), Vischa Shkola, L'vov 1982

13. J. Svoboda and I.J. Corrans: IEEE Trans. Mag. MAG-21 (1985), 53

14. Annon.: Hitachi Ltd. - Development of a new ferromagnet. Mag. Electr. Sep. 3 (1991), 57

Irena Spevakova graduated from the Kharkov State University in 1971 with the M.Sc. degree in physics and joined the Kirensky Institute of Physics, Krasnoyarsk, Russia, where she was engaged in research into magnetic properties of low-dimensional magnetics. She obtained her Ph.d. degree in physics of magnetic phenomena in 1980. Subsequent work in the Kharkov Municipal Research Institute was concerned with the development of technological processes and equipment based on magnetic separation as applied to the purification and recycling of domestic and industrial waste waters and technological liquors. At present, she is working at the Technion, Haifa, Israel. Her research interests include the use of magnetic technology and minerals for environmental protection and magnetic treatment of aqueous solutions for the application in industry and agriculture.

Keywords: magnetic filter, heterogeneous catalyst, Raney nickel, magnetite 